\title{
Characteristics of primary dentition occlusion among preschool children with autism spectrum disorder
}

\begin{abstract}
Objective: To determine the characteristics of primary dentition occlusion among Saudi preschool children with autism spectrum disorder (ASD) and compare it with Saudi preschool children without ASD.

Methods: This study was conducted on a randomly selected sample of 3-6 year old Saudi preschool children in Riyadh, Saudi Arabia. The sample consisted of a study group (SG) of 150 children with ASD and a control group (CG) of age- and gender-matched 150 children without ASD. Clinical examinations to determine primary molar relationship, primary canine relationship, overbite, overjet, posterior crossbite and scissors bite were performed at ASD centers and kindergartens.

Results: Significant differences were found in the occlusal characteristics of the primary dentition between the SG and CG $(\mathrm{p}<0.05)$. Most children in the $\mathrm{SG}$ exhibited a distal step molar relationship (51.3\%), a class II canine relationship (56.7\%), and increased overjet and overbite. Most of the CG children exhibited a flush terminal molar relationship (83.3\%), a class I canine relationship (77.3\%), normal overbite and overjet. The prevalence of posterior crossbite was significantly higher in the $\mathrm{SG}$ than the $\mathrm{CG}(\mathrm{p}=0.012)$.
\end{abstract}

Conclusion: The data revealed that the prevalence of malocclusion was higher in Saudi preschool children with ASD than in non-ASD preschool children.

Keywords: primary dentition occlusion, preschool children, autism spectrum disorder
Volume 9 Issue 4 - 2018

\author{
Ala A Aljubour,' Fares S Al-Sehaibany ${ }^{2}$ \\ 'Postgraduate Student, Department of Pediatric Dentistry \\ and Orthodontics, College of Dentistry, King Saud University, \\ Riyadh, Saudi Arabia \\ ${ }^{2}$ Associate Professor, Department of Pediatric Dentistry and \\ Orthodontics, College of Dentistry, King Saud University, \\ Riyadh, Saudi Arabia
}

\section{Correspondence: Fares S AI-Sehaibany, Department of Pediatric Dentistry and Orthodontics, College of Dentistry, King Saud University, P.O. Box 60169, Riyadh I I545, Saudi Arabia, Tel} +966- I I-46774 I I, Email falsehaibany@ksu.edu.sa

Received: June 20, 2018 | Published: July 05, 2018

\section{Introduction}

Developmental changes in occlusal relationships from the initiation of primary dentition are of considerable clinical interest, and an understanding of these changes forms the basis of the proper development of permanent dentition. ${ }^{1}$ Analysis of the occlusion in primary dentition should consider the anterior and posterior relationship of the maxillary and mandibular arches. ${ }^{2,3}$ Diversity in dental occlusion is the result of a multifactorial hereditary pattern in which genetic and environmental factors play important roles. Malocclusion is primarily due to an imbalance in the developing craniofacial complex. ${ }^{4}$ Certain oral habits performed over a long period of time also may lead to the occurrence of malocclusion. ${ }^{5}$ The early detection and diagnosis of the presence of malocclusion in the primary dentition with proper intervention would play a role in the prevention of malocclusion that are likely to develop in the permanent dentition. ${ }^{6,7}$ The status of occlusion in the primary dentition greatly influences the occlusion of the permanent dentition, but its features differ in diverse populations and ethnic groups. ${ }^{8-12}$ This aspect of primary dentition occlusion has been widely studied in the general populations of various countries, ${ }^{10-14}$, but studies of children with special needs, particularly children with autism spectrum disorder (ASD), are scarce. Autism spectrum disorder is a neurodevelopment and cognitive disorder that is characterized by intellectual impairments in the acquisition of some of the most important life skills, including deficiencies in interpersonal and communication skills, impaired social interactions, and constrained and repetitive behaviors. ${ }^{15,16}$ Autism spectrum disorder is approximately four times more prevalent in males than females, but it is more severe in females. Onset generally occurs during the first three years of life. ${ }^{17,18}$
Epidemiological research on ASD in Arab countries is relatively new due to the associated social stigma, and the burden of ASD remains unclear. One review examined the epidemiology of ASD in Arab gulf countries and estimated a prevalence of ASD of 1.4 to 29 per 10,000 persons, which reveals a serious health concern in this region of the world. ${ }^{19}$ Children with ASD tend to commonly exhibit damaging oral habits, such as bruxism, tongue biting, thumb sucking, picking at the gingiva and object biting in addition to self-injurious behavior. ${ }^{20-22}$ These detrimental oral habits are seriously concerning because these activities may lead to the occurrence of malocclusion. ${ }^{5}$ The purpose of the present study was to determine the characteristics of primary dentition occlusion among Saudi preschool children with ASD (study group=SG) and compare it with Saudi preschool children without ASD (control group $=\mathrm{CG}$ ).

\section{Materials and methods}

This study was conducted on 300 Saudi preschool children aged 3-6 years (150 SG children and 150 age- and gender-matched CG children) who attended private kindergartens and ASD centers in Riyadh, Saudi Arabia. A stratified cluster random sampling was used to select the required sample of SG and CG children. The Research and Ethical Committee of Human Studies at the College of Dentistry Research Center, King Saud University, Riyadh, Saudi Arabia approved the study protocol and consent form. Letters that explained the nature of the study and provided informed parental consent for the child's participation in the study were delivered to parents through the centers' principals. Clinical examinations were performed at the center's facility while the children were seated on a regular chair in an upright position with their teeth in centric occlusion. Occlusal 
evaluation was performed only on children with complete primary dentition and without any erupted permanent teeth or extensive caries that would affect the mesio-distal or occluso-gingival dimension of a tooth. One examiner performed all examinations throughout the entire study. Intra-examiner reliability was established via re-examination of 30 children (15 SG, $15 \mathrm{CG}$ ) on 2 different visits scheduled one week apart (Kappa $=0.87$ ). The following occlusal characteristics were recorded using the Foster and Hamilton criteria: ${ }^{23}$

\section{Second primary molar relationship}

Flush Terminal Plane: The distal surfaces of the upper and lower second primary molars were in the same vertical plane.

Distal Step: The distal surface of the lower primary second molar had a posterior relationship to the distal surface of the upper primary second molar.

Mesial Step: The distal surface of the lower primary second molar had an anterior relationship to the distal surface of the upper primary second molar.

The second primary molar relationship was recorded as a flush terminal plane if one side exhibited a distal or mesial step and the other side exhibited a flush terminal plane. The molar relationship was excluded from the study if one side exhibited a mesial step and the other side exhibited a distal step.

\section{Primary canine relationship}

Class I: The tip of the maxillary primary canine tooth was in the same vertical plane as the distal surface of the mandibular primary canine.

Class II: The tip of the maxillary primary canine tooth was mesial to the distal surface of the mandibular primary canine.

Class III: The tip of the maxillary primary canine was distal to the distal surface of the mandibular primary canine.

The primary canine relationship was recorded as class I if one side was categorized as class II or class III and the other side was categorized as class I. The canine relationship was excluded from the study if one side of the tooth was categorized as class II and the other side was categorized as class III.

\section{Overbite}

The overbite was graded according to the percentage of coverage of the mandibular primary incisors by maxillary primary incisors and recorded as, Normal: Coverage was up to $50 \%$; Increased: Coverage was greater than 50\%; Edge-to-edge: The incisal edge of the maxillary primary incisors were in contact with the incisal edge of the mandibular primary incisors; and Anterior open bite: no vertical overlap was observed between the upper and lower primary incisors.

\section{Overjet}

The overjet was measured in millimeter as the greatest distance between the incisal edges of the maxillary and mandibular primary incisors in the occlusal plane and recorded as:

Normal (0-2mm); Increased ( $>2 \mathrm{~mm})$; and Reversed $(<0 \mathrm{~mm})$.

Posterior Crossbite: This condition was recorded when one or more of the maxillary primary canines or molars occluded lingual to the buccal cusps of the opposing mandibular teeth.

Scissors Bite: This condition was recorded when one or more maxillary primary molars occluded buccal to the buccal surfaces of the corresponding mandibular teeth.

The data were manually entered into the Statistical Package for the Social Sciences software package (IBM, SPSS version 20, Chicago, IL, USA) and analyzed using a significance level of $p<0.05$. Statistical analyses included frequency distribution, Fisher's exact test, and Chisquare test to assess the significance of differences between groups.

\section{Results}

The age of the children in each group ranged from 3 to 6 years with a mean age of $4.9 \pm 0.6$ years for the $S G$ and $4.6 \pm 0.4$ years for the CG. A total of 218 males and 82 females were examined, and the genders were equally distributed in both groups. The SG and CG consisted of $109(72.7 \%)$ male and $41(27.3 \%)$ female preschool children (Table 1). Table 2 shows significant differences in the occlusal characteristics of the primary dentition between the SG and CG. Most children in the SG exhibited a distal step (51.3\%), but the flush terminal molar relationship (83.3\%) was more evident in CG children. The percentage distributions of the different canine relationships revealed that most of the SG children were categorized as class II, and class I was more prominent in CG children. Most SG children exhibited increased overbite and overjet. In contrast, most of the CG children exhibited a normal overbite and overjet. Tables 3 \& Table 4 show no significant differences in the occlusal characteristics of the primary dentition based on gender for the SG and CG, respectively. A total of $13.3 \%$ of SG children exhibited a posterior crossbite, which was significantly higher than the prevalence observed in CG children $(6.0 \%),(p=0.012)$. Unilateral posterior crossbite was the most common type of posterior crossbite in the $\mathrm{SG}$ and $\mathrm{CG}$ and was present in $85 \%$ and $77.7 \%$ of children, respectively. One participant in the SG exhibited a unilateral scissors bite. However, no case of scissors bite was recorded in the CG. In the SG, posterior crossbite exhibited a significantly higher prevalence in females $(34.1 \%)$ than in males $(5.5 \%)(\mathrm{p}=0.001)$. Unilateral posterior crossbite was the most common type of posterior crossbite in both genders and was present in $83.3 \%$ and $85.7 \%$ of males and females, respectively. A unilateral scissors bite was observed in one male child $(0.6 \%)$. However, scissors bite was not recorded in any female children. In the CG, posterior crossbite exhibited a significantly higher prevalence in females (14.6\%) than in males $(2.7 \%)(\mathrm{p}=0.001)$. Unilateral posterior crossbite was the most common type in both genders and was present in $66.6 \%$ and $83.3 \%$ of males and females, respectively. However, no case of scissors bite was recorded in any $\mathrm{CG}$ children.

Table I Gender-wise distribution of the participants in the SG and CG

\begin{tabular}{ccccc}
\hline \multicolumn{2}{c}{ Group } & Male & Female & Total $(\mathrm{N}=300)$ \\
\hline SG & $\mathrm{N}$ & 109 & $4 \mathrm{I}$ & 150 \\
& $\%$ & 72.7 & 27.3 & 100 \\
$\mathrm{CG}$ & $\mathrm{N}$ & 109 & $4 \mathrm{I}$ & 150 \\
& $\%$ & 72.7 & 27.3 & 100 \\
\hline
\end{tabular}


Table 2 Prevalence of occlusal characteristics of the primary dentition among the groups

\begin{tabular}{|c|c|c|c|c|}
\hline \multicolumn{2}{|c|}{ Occlusal characteristics } & \multirow{2}{*}{$\begin{array}{l}\text { SG (N=1 50) N (\%) } \\
53(35.3)\end{array}$} & \multirow{2}{*}{$\begin{array}{l}\text { CG (N=150) N (\%) } \\
125(83.3)\end{array}$} & \multirow[t]{2}{*}{ p-value } \\
\hline & Flush Terminal & & & \\
\hline \multirow[t]{3}{*}{ Molar relationship } & Distal step & $77(51.3)$ & $6(4.0)$ & \multirow[t]{3}{*}{0.001} \\
\hline & Mesial step & $20(13.4)$ & $19(12.7)$ & \\
\hline & Class I & $59(39.3)$ & $116(77.3)$ & \\
\hline \multirow[t]{3}{*}{ Canine relationship } & Class II & $85(56.7)$ & $23(15.4)$ & \multirow[t]{3}{*}{0.001} \\
\hline & Class III & $6(4.0)$ & II (7.3) & \\
\hline & Normal & $43(28.7)$ & $78(52.0)$ & \\
\hline \multirow[t]{4}{*}{ Overbite } & Increased & $68(45.3)$ & $25(16.7)$ & \multirow{2}{*}{0.001} \\
\hline & Edge-to-edge & $5(3.3)$ & $31(20.6)$ & \\
\hline & Anterior open bite & $34(22.7)$ & $16(10.7)$ & \\
\hline & Normal & $54(36.6)$ & III (74.0) & \\
\hline \multirow[t]{2}{*}{ Overjet } & Increased & $90(59.3)$ & $34(22.7)$ & \multirow[t]{2}{*}{0.001} \\
\hline & Reversed & $6(4.1)$ & $5(3.3)$ & \\
\hline
\end{tabular}

Table 3 Gender-wise distribution of the occlusal characteristics of the primary dentition for the SG

\begin{tabular}{lllll}
\hline Occlusal characteristics & & Male $(\mathbf{N}=1$ 09) $\mathbf{N}(\%)$ & Female (N=4I) N (\%) & p-value \\
\hline \multirow{3}{*}{ Molar relationship } & Flush Terminal & $37(33.9)$ & $16(39.0)$ & \\
& Distal step & $58(53.2)$ & $19(46.4)$ & 0.135 \\
& Mesial step & $14(12.9)$ & $6(14.6)$ & \\
Canine relationship & Class I & $42(38.5)$ & $17(41.5)$ & 0.257 \\
& Class II & $63(57.8)$ & $22(53.7)$ & \\
Class III & $4(3.7)$ & $2(4.8)$ & \\
Overbite & Normal & $31(28.4)$ & $12(29.3)$ & 0.173 \\
& Increased & $49(45.0)$ & $19(46.4)$ & \\
& Edge-to-edge & $3(2.8)$ & $2(4.8)$ & \\
Overjet & Anterior open bite & $26(23.8)$ & $8(19.5)$ & \\
& Normal & $39(35.8)$ & $15(36.9)$ & 0.268 \\
\hline
\end{tabular}

Table 4 Gender-wise distribution of the occlusal characteristics of the primary dentition for the CG.

\begin{tabular}{|c|c|c|c|c|}
\hline Occlusal characteristics & & Male (N=109) N (\%) & Female (N=4I) N (\%) & p-value \\
\hline & Flush Terminal & $90(82.6)$ & $35(85.4)$ & \multirow{3}{*}{0.128} \\
\hline \multirow[t]{3}{*}{ Molar relationship } & Distal step & $5(4.6)$ & $\mathrm{I}(2.4)$ & \\
\hline & Mesial step & 14 (I2.8) & $5(12.2)$ & \\
\hline & Class I & $83(76.1)$ & $33(80.6)$ & \multirow{3}{*}{0.452} \\
\hline \multirow[t]{2}{*}{ Canine relationship } & Class II & $18(16.6)$ & $5(12.2)$ & \\
\hline & Class III & $8(7.3)$ & $3(7.2)$ & \\
\hline \multirow{3}{*}{ Overbite } & Normal & $57(52.3)$ & $2 \mid(5 \mid .2)$ & \multirow{4}{*}{0.567} \\
\hline & Increased & $18(16.5)$ & $7(17.0)$ & \\
\hline & Edge-to-edge & $22(20.2)$ & $9(21.9)$ & \\
\hline \multirow{4}{*}{ Overjet } & Anterior open bite & $12(11.0)$ & $4(9.9)$ & \\
\hline & Normal & $80(73.3)$ & $31(75.0)$ & \multirow{3}{*}{0.484} \\
\hline & Increased & $25(22.8)$ & $9(22.5)$ & \\
\hline & Reversed & $4(3.9)$ & I (2.5) & \\
\hline
\end{tabular}




\section{Discussion}

This study contributes important baseline information on the occlusal characteristics of the primary dentition in Saudi preschool children with ASD compared to their healthy counterparts. An understanding of anterior and posterior primary dentition occlusion is crucial for clinicians to plan early interceptive treatment. Early treatment would likely reduce the percentage of permanent tooth extractions and the overall treatment period as well as increase to the likelihood of obtaining better aesthetic and functional results. ${ }^{24}$ Children without early intervention endure aesthetic, functional, phonetic and psycho-social problems caused by malocclusion, which negatively affect their childhood. ${ }^{7}$ Most SG children exhibited a distal step, but the flush terminal plane predominated in CG children. A distal molar relationship is likely to be present in the permanent dentition when the primary dentition exhibits a distal step. ${ }^{25}$ This relationship is relevant to the clinician because self-correction would not be possible, and early orthodontic intervention would be required.

The finding that most CG children exhibited a class I canine relationship, followed by class II and class III relationships, is consistent with previous studies. ${ }^{8,10,26-28}$ In contrast, most SG children exhibited a class II canine relationship, followed by class I and class III relationships, which is a novel finding in children with ASD. The prevalence of posterior crossbite in the present study was significantly higher in the SG $(13.3 \%)$ than in the CG $(6.0 \%)$. However, previous studies $^{8-10,27}$ have reported a higher prevalence of posterior crossbite in CG children, which contrasts the present findings. The overall prevalence of scissors bite was only $0.6 \%$ in SG children, and this finding is consistent with previous studies of non-ASD children in various countries. ${ }^{9,10,14,26} \mathrm{Few}$ studies have compared gender and occlusal relationships with regard to the occlusion characteristics of the primary dentition. Most of these studies revealed no significant differences between genders or occlusal relationships for the primary molar or primary canine occlusal relationships. ${ }^{6,9,27-29}$ These findings are consistent with our study because we found no significant differences in gender and the molar and canine occlusal relationships between CG and SG children. In contrast, an Indian study revealed significant differences between male and female occlusal characteristics. ${ }^{26}$ Our study revealed that increased overjet $(59.3 \%)$ was the most common observed type of overjet in the SG which is higher than the findings of other studies of non-ASD children. ${ }^{6,10,13}$ The difference was most likely due to the abnormal oral habits in children with ASD. Certain limitations associated with the results of this study merit mention. First, a lack of previously published studies using a similar study design and specific cohorts, such as children with special needs, makes comparisons of all aspects of the results difficult. Other aspects, such as nutritional and behavioral factors, were not compared. A follow-up study would help elucidate the changes in occlusion in the permanent dentition and determine the impact of primary dentition occlusion on these changes.

\section{Conclusion}

The present study provides previously unavailable data on the occlusal characteristics in the primary dentition of preschool children with ASD compared to non-ASD children in Riyadh, Saudi Arabia. The results of this study emphasize the importance of paying special attention to occlusion of primary dentition because timely diagnosis and treatment may aid in the prevention of future complications.

\section{Acknowledgements}

The authors thank the College of Dentistry Research Center and The Deanship of Graduate Studies at King Saud University, Riyadh, Saudi Arabia, for supporting this study.

\section{Conflict of interest}

The authors declare that there is no conflict of interest.

\section{References}

1. Aznar T, Galan AF, Marin I, et al. Dental arch diameters and relationships to oral habits. Angle Orthod. 2006;76(3):441-5.

2. Alonso Chevitarese AB, Valle DD, Moreira TC. Prevalence of malocclusion in 4-6 year old Brazilian children. $J$ Clin Pediatr Dent. 2003;27(1):81-5.

3. Andlaw R, Rock W. A manual of paediatric dentistry: Elsevier Health Sciences; 1996.

4. Kawala B, Antoszewska J, Nęcka A. Genetics or environment? A twinmethod study of malocclusions. World J Orthod. 2007;8(4):405-10.

5. Dos Santos RR, Nayme J, Garbin A, et al. Prevalence of malocclusion and related oral habits in 5-to 6-year-old children. Oral Health Prev Dent. 2012;10(4):311-8.

6. Moslemi M, Nadalizadeh S, Sarsanghizadeh S. Evaluation of dental occlusion in 3-5 year-old children. Int J Environ Res Public Health. $2015 ; 1: 48-53$

7. Kirzioglu Z, Simsek S, Yilmaz Y. Longitudinal occlusal changes during the primary dentition and during the passage from primary dentition to mixed dentition among a group of Turkish children. Eur Arch Paediatr Dent. 2013;14(2):97-103.

8. Abu Alhaija ES, Qudeimat MA. Occlusion and tooth/arch dimensions in the primary dentition of preschool Jordanian children. Int J Paediatr Dent. 2003;3(4):230-9.

9. Otuyemi O, Sote E, Isiekwe M, et al. Occlusal relationships and spacing or crowding of teeth in the dentitions of 3-4-year-old Nigerian children. Int J Paediatr Dent. 1997;7(3):155-60.

10. Farsi NM, Salama FS. Characteristics of primary dentition occlusion in a group of Saudi children. Int J Paediatr Dent. 1996;6(4):253-9.

11. Jones M, Mourino A, Bowden T. Evaluation of occlusion, trauma, and dental anomalies in African-American children of metropolitan Headstart programs. J Clin Pediatr Dent. 1993;18(1):51-4.

12. Kerosuo H. Occlusion in the primary and early mixed dentitions in a group of Tanzanian and Finnish children. ASDC J Dent Child. 1990;57(4):293-8.

13. Biria M, Eslami Amirabadi G, Rahmani M. Evaluation of occlusion in 3-5 year old nurseryschools children in Tehran-2003. Shahid Beheshti University Dent J. 2004;21(4):661-70.

14. Tschill P, Bacon W, Sonko A. Malocclusion in the deciduous dentition of Caucasian children. Eur J Orthod. 1997;19(4):361-7.

15. Kim SK. Recent update of autism spectrum disorders. Korean J Pediatr. 2015;58(1):8-14.

16. APA. Diagnostic and Statistical Manual of Mental Disorders. DSM-5. Atlington, VA. American Psychiatric Association; 2013.

17. Weddell JA, Sanders BJ, Jones JE. Dental problems of children with special health care needs. In: McDonald and Avery's Dentistry for the Child and Adolescent (Tenth Edition): Elsevier; 2016:513-39. 
18. Friedlander A, Yagiela J, Paterno V, et al. The pathophysiology, medical management, and dental implications of autism. J Calif Dent Assoc. 2003;31(9):681-691.

19. Salhia HO, Al-Nasser LA, Taher LS, et al. Systemic review of the epidemiology of autism in Arab Gulf countries. Neurosciences. 2014;19(4):291-6.

20. Medina A, Sogbe R, Gómez-Rey A, et al. Factitial oral lesions in an autistic paediatric patient. Int J Paediatr Dent. 2003;13(2):130-7.

21. Al-Sehaibany FS. Occurrence of oral habits among preschool children with Autism Spectrum Disorder. Pak J Med Sci. 2017;33(5):1156-60.

22. Murshid EZ. Oral health status, dental needs, habits and behavioral attitude towards dental treatment of a group of autistic children in Riyadh, Saudi Arabia. Saudi Dent J. 2005;17(3):132-139.

23. Foster TD, Hamilton MC. Occlusion in the primary dentition. Study of children at 2 and one-half to 3 years of age. Br Dent J. 1969;126(2):76-9.

24. Ngan P, Fields H. Orthodontic diagnosis and treatment planning in the primary dentition. ASDC J Dent Child. 1995;62(1):25-33.
25. Bishara SE, Jakobsen JR, Treder J, et al. Arch length changes from 6 weeks to 45 years. Angle Orthod. 1998;68(1):69-74.

26. Bhayya DP, Shyagali TR. Gender influence on occlusal characteristics of primary dentition in 4-to 6-year-old children of Bagalkot City, India. Oral Health Prev Dent. 2011;9(1):17-27.

27. De Almeida ER, Narvai PC, Frazão P, et al. Revised criteria for the assessment and interpretation of occlusal deviations in the deciduous dentition: a public health perspective. Cad Saude Publica. 2008;24(4):897-904.

28. Yilmaz Y, Gurbuz T, Simsek S, et al. Primary canine and molar relationships in centric occlusion in three to six year-old Turkish children: a cross-sectional study. J Contemp Dent Pract. 2006;7(3):59-66.

29. Khan R, Singh N, Govil S, et al. Occlusion and occlusal characteristics of primary dentition in North Indian children of East Lucknow region. Eur Arch Paediatr Dent. 2014;15(5):293-9. 